\title{
Long-term follow-up study of gastric adenoma; tumor-associated macrophages are associated to carcinoma development in gastric adenoma
}

\author{
Daiki Taniyama ${ }^{1,2,3} \cdot$ Kiyomi Taniyama $^{1,2,3}$ (D) Kazuya Kuraoka ${ }^{2,3} \cdot$ \\ Junichi Zaitsu ${ }^{2,3} \cdot$ Akihisa Saito $^{2,3} \cdot$ Hirofumi Nakatsuka $^{4} \cdot$ Naoya Sakamoto $^{1}$. \\ Kazuhiro Sentani $^{1} \cdot$ Naohide Oue $^{1} \cdot$ Wataru Yasui $^{1}$
}

Received: 13 October 2016/Accepted: 4 March 2017/Published online: 20 March 2017

(c) The International Gastric Cancer Association and The Japanese Gastric Cancer Association 2017

\begin{abstract}
Background Some gastric adenomas may progress to adenocarcinoma in a short time, but others remain as adenoma for a long time.

Methods Among 1138 cases diagnosed as adenoma by biopsy at Kure Medical Association Hospital between 1990 and 2010, 51 adenomas were enrolled. Of these, 28 adenomas (group A) were followed for 60 months or longer with no progression to adenocarcinoma within 60 months, and the other 23 adenomas (group B) were upgraded to carcinoma by consecutive biopsies performed within 1 year after the first biopsy. These adenomas were compared clinicopathologically and immunohistochemically.

Results Macroscopically, the mean size of group B adenomas was significantly larger than that of group A adenomas (18.6 vs. $9.9 \mathrm{~mm}$ ) at the first biopsy. The frequency of a depressed area in the adenoma was significantly higher in group B than group A. Microscopically none of group A
\end{abstract}

Electronic supplementary material The online version of this article (doi:10.1007/s10120-017-0713-x) contains supplementary material, which is available to authorized users.

Kiyomi Taniyama

taniyamak@kure-nh.go.jp

1 Department of Molecular Pathology, Hiroshima University Institute of Biomedical and Health Sciences, Hiroshima, Japan

2 Institute for Clinical Research, National Hospital Organization, Kure Medical Center and Chugoku Cancer Center, 3-1 Aoyama-cho, Kure 737-0023, Japan

3 Department of Diagnostic Pathology, National Hospital Organization, Kure Medical Center and Chugoku Cancer Center, Kure, Japan

4 Department of Surgery, Kure Medical Association Hospital, Kure, Japan but 7 (30.4\%) of 23 group B adenomas showed severe atypia. Each of a highly proliferative gland measured by Ki-67 labeling, cellular atypical grade, gastric phenotype defined by MUC5AC and MUC6 and CD204-positive tumor-associated macrophage (TAM) was a significant risk factor for adenocarcinoma development in gastric adenoma by univariate analysis. Only moderate or severe atypia of adenoma cells and the TAM number in the stroma of adenomas were independent risk factors by multivariate analysis.

Conclusions As independent risk factors, cellular atypia may reconfirm the importance of morphological analysis, and the TAM number may indicate the significance of TAM function in gastric adenoma.

Keywords Gastric adenoma - Adenoma-carcinoma sequence $\cdot$ Tumor-associated macrophages

\section{Introduction}

The World Health Organization (WHO) defines gastric adenomas as polypoid lesions composed of tubular and/or villous structures lined by dysplastic epithelium [1, 2]. They are divided into two grades, low or high. Adenomas with mild to moderate atypia are classified into low-grade, and with severe atypia considered high grade. They occur spontaneously or in the context of familial adenomatous polyposis (FAP). The prevalence of gastric adenomas is reported at between 0.65 and $3.75 \%$ of healthy people in western countries and 9-72\% in Asia [3-5]. Gastric adenoma can occur anywhere in the stomach, but most commonly in the antrum [6]. Most are found incidentally during endoscopic screening examinations. 
Several studies have reported that most adenomas have an intestinal phenotype and only a few adenomas have a gastric phenotype [7]. Although gastric adenoma is regarded as a benign epithelial tumor, some adenomas progress to carcinoma in a short time, whereas others remain as adenoma for a long time [8]. Although an adenoma-carcinoma sequence has been speculated as a course of carcinogenesis in gastric intestinal-type carcinoma, estimates vary. This uncertainty arises from a wide difference in diagnostic criteria $[9,10]$, the selected population, followup period, etc. Small biopsied specimens may sometimes result in misdiagnosis as adenomas that have synchronous adenocarcinoma in parts. Only a few studies have done long histological and immunohistochemical follow-up studies to evaluate gastric adenoma and its pathological and clinical significance $[11,12]$.

Previous studies have suggested that depressed type, larger size, surface appearance with erythema, unevenness, ulcer or erosion, and a villous structure on biopsy specimens are prognostic factors for an upgraded diagnosis in patients with low-grade adenoma [13]. The risk of malignant transformation increases with pathological grade. The rate of malignant change of low-grade adenoma has been reported as between 3-9 and 60-85\% for high-grade adenoma $[10,12,14-18]$. These previous studies focused only at the point when diagnosis of carcinoma was made and not from early on. We tried to clarify the malignant potential of adenomas by focusing on an earlier time point. A long observation period may provide new knowledge about which time point is important to focus on.

The molecular characteristics of adenoma, especially of the biopsy specimen, have not been fully elucidated. Currently, there is no biomarker that distinguishes an adenoma with malignant potential from immutable adenoma. Cancer stem cells (CSCs) are known to promote cancer metastasis, chemoresistance and relapse in a variety of cancers [19]. Little has been reported on the role of CSCs in an adenoma-carcinoma sequence. Tumor-associated macrophages (TAMs) have been reported in tumorigenesis, angiogenesis, matrix remodeling and metastasis in cancer tissues [20,21]. They have a prognostic impact on cancers of the breast, lung, prostate and pancreas [22-25] . However, the role of TAM in gastric adenoma is not known.

In the current study, biopsy samples were obtained at Kure Medical Association Hospital. Patients were identified from a private clinic in Kure City and were followed up by these private clinic physicians. These physicians tend to follow-up on gastric adenoma once or twice in 1 year while it is a benign lesion. Patients were Kure City citizens and would not move to other hospitals. The characteristics of these physicians and patients facilitated long-term follow-up in the present study. We compared adenomas that remained as adenoma for a long time with adenomas that progressed to carcinoma in a short time and identified risk factors of adenomas that progress to adenocarcinoma in a short time or that harbor adenocarcinoma synchronously.

\section{Materials and methods}

\section{Adenomas}

Among 1138 biopsied cases diagnosed as tubular adenoma at Kure Medical Association Hospital between 1990 and 2010, 51 adenomas were enrolled. The patients were 31 males and 20 females. The age of the patients at the time of the initial diagnosis ranged from 48 to 92 years old, with a mean age of 70 years. Twenty-eight adenomas (group A) were followed for 60 months or longer with no progression to adenocarcinoma within 60 months. Twenty-three adenomas (group B) were upgraded to carcinoma by a consecutive biopsy performed within 1 year after the first biopsy. Adenomas of the two groups were compared clinicopathologically and immunohistochemically. The study protocol was approved by the Institutional Review Board of Kure Medical Association Hospital.

\section{Evaluation of clinicopathological features}

The clinicopathological features of the adenomas were investigated retrospectively from endoscopic and pathological records: gender, age at the initial biopsy, follow-up period, size, location, shape, atypical grade and morphological change. Twenty-five adenomas were measured by physicians, but others had no information about size in the endoscopic records. All adenomas were stratified into two groups: smaller than $10 \mathrm{~mm}$ or not according to the schema on the endoscopic records. The stomach was anatomically divided into three portions: upper (U), middle (M) and lower (L) [26].

\section{Evaluation of pathological features}

All adenomas were examined macro- and microscopically through endoscopic biopsy. Follow-up biopsy specimens were taken from the same region where the gastric adenoma had initially been diagnosed and confirmed by macroscopic figures through endoscopic observation (Fig. 2). Biopsy specimens were fixed in a $10 \%$ buffered formalin briefly after the tissue was taken and embedded in paraffin. Hematoxylin and eosin-stained specimens of 3-4 $\mu \mathrm{m}$ thickness were prepared in a routine procedure. Pathological diagnoses were based on the 14th edition of the Japanese Classification of Gastric Carcinoma and the 4th edition 
of the WHO Classification of Tumors of the Digestive System. Diagnosis of low grade was made for mild to moderate cellular atypia, with larger than normal nuclei, elongated and stratified, but stratification was not higher than the basal 1/2 of the cells. Architecturally, low-grade adenomas have tubules of relatively uniform shape and size. Diagnosis of high grade was made with more pleomorphic nuclei and stratification closer to full thickness; there was more variation in cell size and shape. Architectural disorganization comes along with great variation in tubule size. The diagnosis of carcinoma was made only when cellular/nuclear atypia or glandular structures demonstrated a histological appearance sufficient for the diagnosis [27]. When there were several biopsy specimens from the same tumor, the strongest atypical grade was regarded as its diagnosis. Original diagnoses were made by one authorized pathologist (KT) with no immunohistochemical analysis. For the present study, each biopsy specimen was reviewed separately by two authorized pathologists (KK and KT) who had no information about the original diagnosis with an interval of at least 10 years from the original diagnosis.

\section{Antibodies and immunohistochemistry}

Continuous 3-4- $\mu \mathrm{m}$-thick slides were used for immunohistochemical study as reported previously [28]. The antibodies for Ki-67 (clone MIB-1, monoclonal, diluted 1:50; DAKO, Glostrup, Denmark), p53 (clone DO-7, monoclonal, diluted 1:50; DAKO), CD10 (clone 56C6, monoclonal, diluted 1:100; Novocastra, Newcastle, UK), MUC2 (clone Ccp58, monoclonal, diluted 1:100; Novocastra), MUC5AC (clone CLH2, monoclonal, diluted 1:100; Novocastra), MUC6 (clone CLH5, monoclonal, diluted 1:200; Novocastra), CD204 (clone SRA-E5, monoclonal, diluted 1:200; Transgenic, Tokyo, Japan), ALDH1 (clone 44/ALDH, monoclonal, diluted 1:400; BD Biosciences; San Diego, CA, USA), CD44 (clone DF1485, monoclonal, diluted 1:800; Novocastra) and Olfactomedin 4, which were kindly provided by Prof. Wataru Yasui (Department of Molecular Pathology, Hiroshima University Institute of Biomedical and Health Sciences, Hiroshima, Japan) [29], were used as the primary antibodies to detect corresponding protein expression. Immunohistochemistry (IHC) for Ki-67, p53, CD10, MUC2, MUC5AC, MUC6 and Olfactmedin 4 was performed using a VENTANA BenchMark XT automated staining instrument (Ventana), according to the manufacturer's instructions. IHC for CD204, ALDH1 and CD44 was performed using an Autostainer (DAKO), according to the manufacturer's instructions. Very small specimens included in some biopsy cases were excluded from the evaluation of IHC.

\section{Evaluation of immunohistochemistry}

Only unequivocal nuclear staining with p53, cytoplasmic staining with ALDH1 and Olfactmedin 4 and membranous staining with $\mathrm{CD} 44$ antibody were considered positive and classified according to the percentage of stained adenoma cells. A result was considered positive when at least $10 \%$ of the cells were stained. To evaluate the proliferative activity of adenoma, Ki-67-positive nuclei were counted microscopically with a $40 \times$ objective lens among all adenoma cells in the biopsy specimens, and they were expressed as Ki-67 labeling indices (LIs). There was inaccuracy in counting nuclei or cell numbers due to overlap in some areas, and estimation of Ki-67 LI occurred. As such, estimated Ki-67 LIs were adjusted to the Ki-LIs categorized by every $10 \%$ from 10 to $100 \%$. Adenomas were classified into two subgroups: high LI adenomas, for which the Ki-67 LI was $50 \%$ or larger, and low LI adenomas, for which the Ki-67 LI was less than $50 \%$. We defined an adenomatous gland containing more than $80 \%$ of $\mathrm{Ki}-67$ positive cells as a highly proliferative gland and adenomas that contain ten or more highly proliferative glands as highly proliferative adenomas (HPA). Ki-67 LIs were calculated independently by two pathologists (KK and KT), and consensus interpretation was made when there were discordant results.

TAMs were identified as CD204-positive cells having oval to round nuclei with strong membranous/cytoplasmic, but no nuclear staining. TAM numbers in three hot spots were counted by the above-mentioned two pathologists independently using a $40 \times$ objective microscopic lens. The area of adenoma stroma was measured using a DP27 digital camera (Olympus, Tokyo, Japan) and software.

\section{Phenotypic analysis of adenomas}

Phenotypes were classified into four types: gastric (G), intestinal (I), gastric and intestinal mixed (GI) and unclassified $(\mathrm{N})$. Gastric adenomas in which more than $10 \%$ of the cells displayed at least one gastric epithelial cell marker (MUC5AC or MUC6) or intestinal epithelial cell marker (MUC2 or CD10) were classified as a G or I phenotype, respectively. Those that showed both $G$ and I phenotypes were classified as a GI phenotype, and those lacking both phenotypes were classified as a $\mathrm{N}$ phenotype [29].

\section{Statistical methods}

Correlations between clinicopathological items and IHC results for each stain were analyzed by chi-squared test. Univariate and multivariate analysis of risk factors for 
a

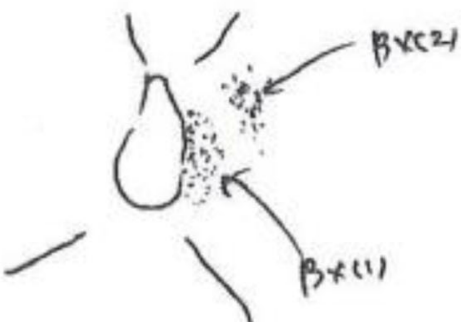

White colored small elevated lesion next to the pyloric ring.

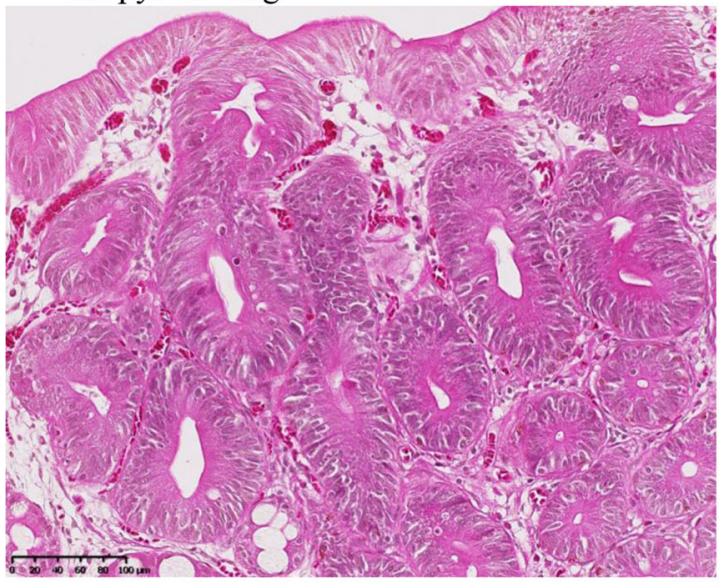

c

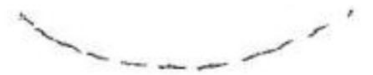<smiles>CC1=C(C)C(C)(C)C(C)=C1C</smiles>

White colored lesion with no change.

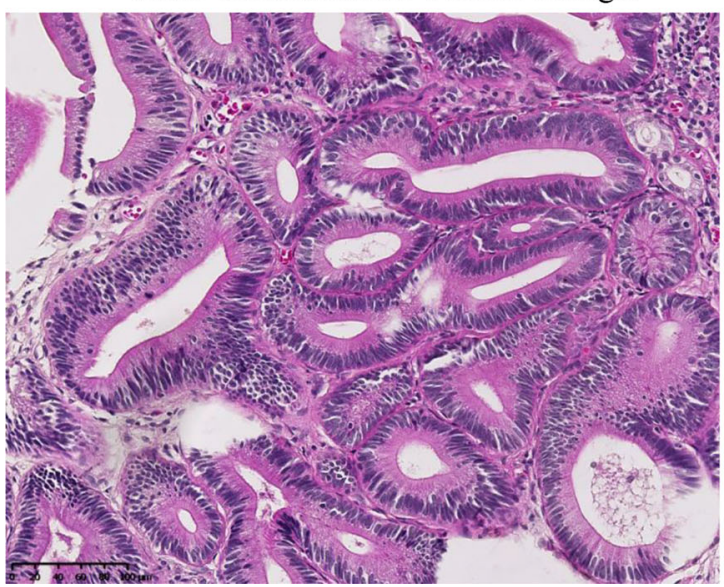

b

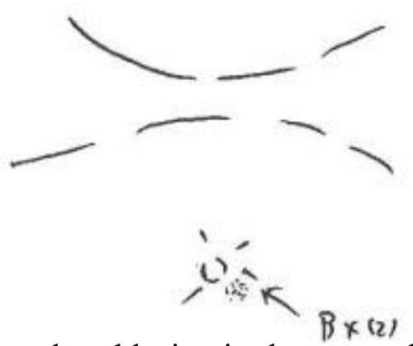

White colored lesion in the same place became unclear.

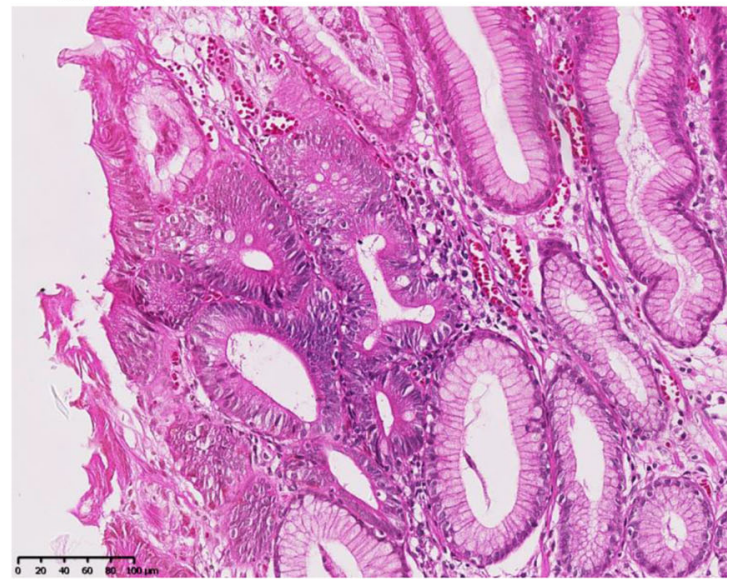

d

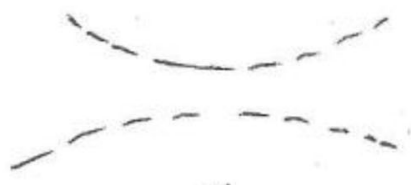

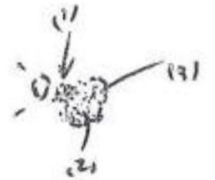

White colored lesion with no change.

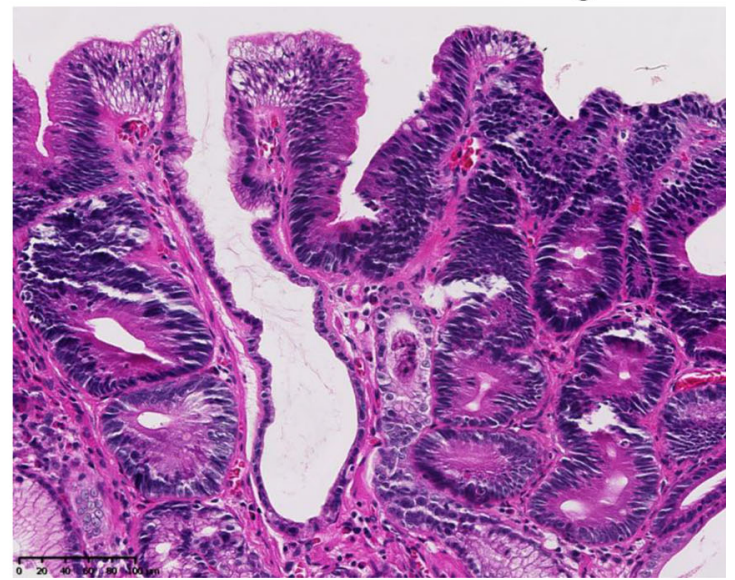

Fig. 1 A case of low-grade adenoma followed for 17 years (A14). Endoscopic records and microscopic view. Three years (a), six years (b), 10 years (c) and 12 years (d) after the initial biopsy

adenocarcinoma were done using a logistic regression analysis model. Risk factors at multivariate analysis were selected by the stepwise method. The odds ratio (OR) and
95\% confidence interval (CI) were estimated from the logistic regression analysis. Statistical analyses were performed with JMP v. 9 (SAS Institute, Cary, NC, USA). In 


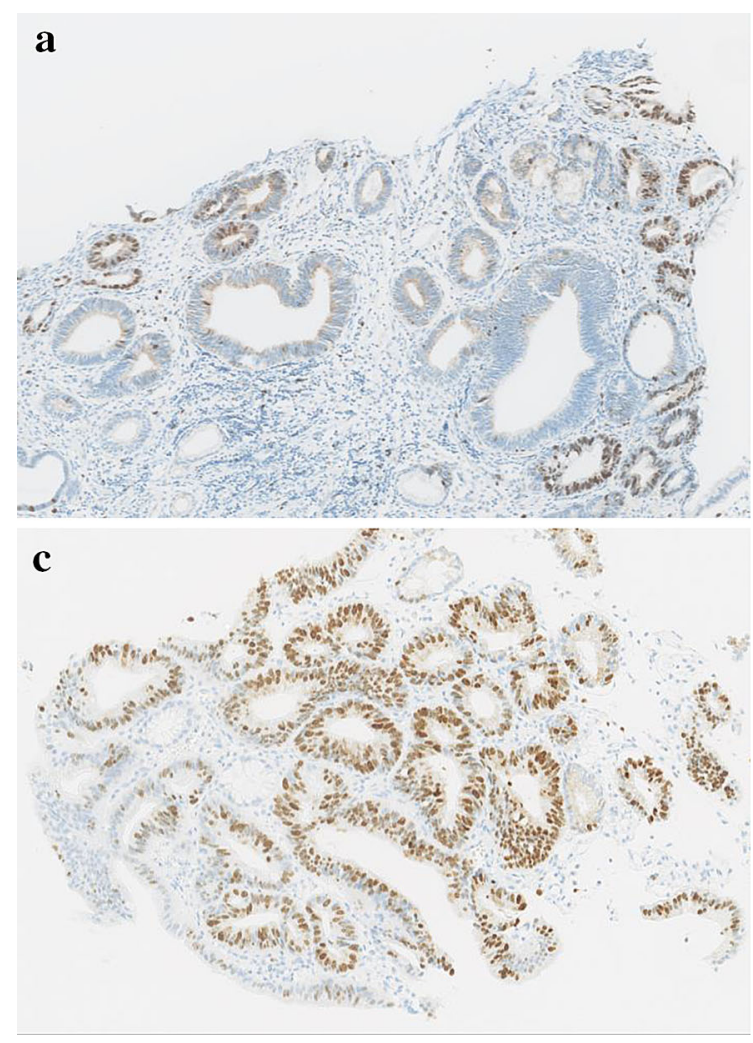

Fig. 2 Immunohistochemical analysis of Ki-67. a, b Low grade adenoma with scattered Ki-67-positive proliferative cells (B8). Although Ki-67 LI was $30 \%$, it contained a lower number of highly

univariate and multivariate analyses, age was treated as a categorical variable (70 years or more vs. less than 70 years). A $p$ value of less than 0.05 was considered statistically significant.

\section{Results}

\section{Clinical and histopathological findings}

Schematic figures of the follow-up data for all adenoma cases is illustrated in Supplementary Fig. 1 (group A) and Supplementary Fig. 2 (group B). Figure 1 shows the macroscopic and microscopic reports at (a) 3, (b) 6, (c) 10 and (d) 12 years after the first examination of A4 adenoma, in which well-differentiated adenocarcinoma developed after about 20 years of follow-up. This adenoma at the first endoscopic examination looked whitish and was described as a 7-mm-sized Yamada polyp-I, flat elevated type, which was located in the posterior wall of the pylorus area (L portion). A depressed lesion was observed at the second biopsy taken after 7 months. Endoscopic follow-up continued until the 213rd month when adenocarcinoma emerged, and it was followed by

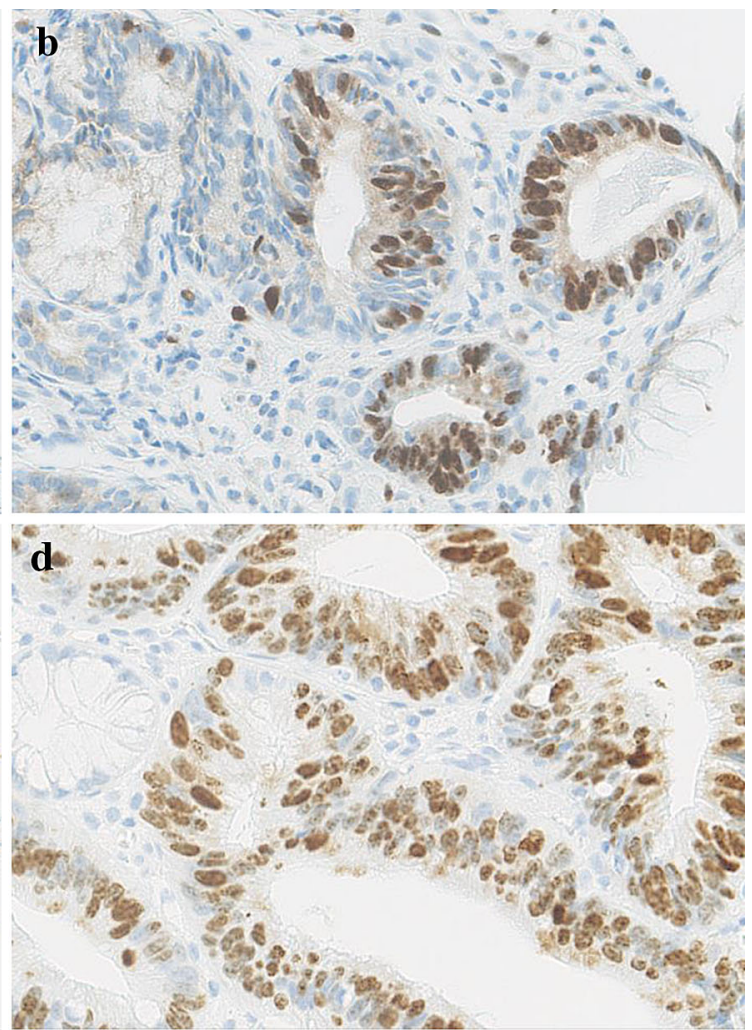

proliferative glands. c, d Highly proliferative adenoma. Proliferating cells positive for Ki-67 are seen diffusely (A11). Ki-67 LI was $80 \%$

distal gastrectomy performed at 218th months. In total, biopsy was performed 25 times before adenocarcinoma developed, and all adenomas were assessed as low grade with mild nuclear atypia. Not only A4, but also A9 developed adenocarcinoma after a long-term follow-up (about 20 years). Both of the resected lesions were intramucosal differentiated type adenocarcinomas surrounded by tubular adenoma.

Table 1 shows clinicopathological features of both groups and their adenomas at the first biopsy. There was no significant difference in age at the first biopsy. On average, 1.73 biopsy samples were taken from one tumor with no statistical difference between the two groups. The mean adenoma sizes of 14 group $\mathrm{A}$ adenomas and 11 group B adenomas were 9.9 and $18.6 \mathrm{~mm}$ in size, respectively, with a significant difference $(p=0.02)$. They were followed for 3212 days on average (1934-7117 days) in group A and for 69 days on average (5-323 days) in group B adenomas. A macroscopic depressed area was found in $2(7.1 \%)$ of 28 group A and in $7(30.4 \%)$ of 23 group B adenomas $(p=0.03)$. Microscopic severe nuclear atypia was only found in group B adenomas $(7 / 23,30.4 \%)$ with a statistical difference $(p<0.0001)$. 
Table 1 Comparison of clinical and histopathological findings of two groups at the initial biopsy $(n=51)$

\begin{tabular}{|c|c|c|c|}
\hline Patient characteristics & $\begin{array}{l}\mathrm{A} \\
(n=28)\end{array}$ & $\begin{array}{l}\text { B } \\
(n=23)\end{array}$ & $p$ value \\
\hline \multicolumn{4}{|l|}{ Sex } \\
\hline Male & $16(57.1 \%)$ & $15(65.2 \%)$ & \multirow[t]{2}{*}{0.56} \\
\hline Female & $12(42.9 \%)$ & $8(34.8 \%)$ & \\
\hline \multicolumn{4}{|c|}{ Age (years) at the first Dx } \\
\hline Mean \pm SD & $68.7 \pm 8.0$ & $73.8 \pm 11.2$ & \multirow[t]{3}{*}{0.06} \\
\hline \multicolumn{3}{|l|}{ Follow-up period } & \\
\hline Mean (range) & $3.212(1.934-7.117)$ days & 69 (5-323) days & \\
\hline \multicolumn{4}{|c|}{ Total number of biopsies performed for making the diagnosis of adenoma } \\
\hline Mean (range) & $8.5(3-26)$ times & $1.1(1-2)$ times & \\
\hline \multicolumn{4}{|c|}{ Tumor size at the first $\mathrm{Dx}$} \\
\hline Mean $\pm \mathrm{SD}(\mathrm{mm})$ & $9.9 \pm 7.4(n=14)$ & $18.6 \pm 9.92(n=11)$ & 0.02 \\
\hline $10 \mathrm{~mm}$ & $19(67.9 \%)$ & $8(34.8 \%)$ & \multirow[t]{2}{*}{0.02} \\
\hline$>11 \mathrm{~mm}$ & $9(32.1 \%)$ & $15(65.2 \%)$ & \\
\hline \multicolumn{4}{|l|}{ Location } \\
\hline $\mathrm{U}$ & $5(17.9 \%)$ & $3(8.7 \%)$ & \multirow{3}{*}{0.4} \\
\hline M & $13(46.4 \%)$ & $8(34.8 \%)$ & \\
\hline $\mathrm{L}$ & $10(35.7 \%)$ & $12(52.2 \%)$ & \\
\hline \multicolumn{4}{|l|}{ Morphological change } \\
\hline Present & $11(39.3 \%)$ & $4(17.4 \%)$ & \multirow[t]{2}{*}{0.08} \\
\hline Absent & $17(60.7 \%)$ & $19(82.6 \%)$ & \\
\hline \multicolumn{4}{|l|}{ Depressed lesion } \\
\hline Present & $2(7.1 \%)$ & $7(30.4 \%)$ & \multirow[t]{2}{*}{0.03} \\
\hline Absent & $26(92.9 \%)$ & $16(69.6 \%)$ & \\
\hline \multicolumn{4}{|l|}{ Atypia } \\
\hline Severe & 0 & $7(30.4 \%)$ & \multirow{3}{*}{$<0.0001$} \\
\hline Moderate & $4(14.3 \%)$ & $13(56.5 \%)$ & \\
\hline Mild & $24(85.7 \%)$ & $3(13.0 \%)$ & \\
\hline
\end{tabular}

Statistically significant values are shown in bold

$D x$ diagnosis, $U$ upper, $M$ middle, $L$ lower parts

\section{Immunohistochemical analysis of adenomas}

To further validate the characteristics of each group, we performed IHC for Ki-67 and p53. As for p53 IHC, only two of group A and three of group B adenomas showed focal or diffuse nuclear positivity. In other adenomas, p53 positivity was observed sparsely, and there was no significant relation between p53 IHC and nuclear atypical grade or adenocarcinoma development. We also evaluated CSC markers, such as ALDH1 and CD44, and Olfactomedin 4, which are known as cancer-specific genes in gastric cancer tissue [30]. All of them showed no significant difference between the two groups (data not shown). Mean Ki-67 LIs were 31.4\% in group A and $39.0 \%$ in group B adenomas with no statistical difference. However, high Ki-67 LI adenomas and HPA were more frequently detected in group B than group A adenomas (high Ki-67LI $p=0.02$; HPA $p=0.04$; Fig. 2; Table 2). In A4 and A9 adenomas, Ki-67 LI increased gradually during follow-up (data not shown).
As for mucin phenotypes, group A adenomas tended to show the I phenotype $(p=0.03)$ and group B adenomas the $\mathrm{G}$ phenotype ( $p=0.02$; Fig. 3; Table 2). These data indicated that evaluation of mucin phenotypes in adenoma can be useful for detecting high-risk adenomas, and this was consistent with previous reports [7].

\section{TAM number in gastric adenomas}

Mean TAM numbers were 21.8 in group A and 49.9 in group B adenomas with a statistical difference as well as mean TAM numbers per $1 \mathrm{~mm}^{2}(p<0.0001$, Fig. 4 and Table 2). Areas of stroma measured in adenoma were 7.0 and $6.9 \times 10^{4} \mu \mathrm{m}^{2}$, respectively, with no statistical difference. In A4 adenoma, the TAM numbers in the areas of adenoma stroma at initial biopsy, 70, 121 and 199 months were $24,23,60$ and 40, respectively, while adenoma remained low grade. In A9 adenoma, the TAM numbers at initial biopsy, 74, 128 and 175 months were 11, 16, 58 and 
Table 2 Comparison of immunohistochemical findings between group A and group B

\begin{tabular}{|c|c|c|c|c|c|}
\hline & \multicolumn{2}{|l|}{ Group } & \multirow[t]{2}{*}{$p$ value } & \multirow[t]{2}{*}{ Average number of TAMs } & \multirow[t]{2}{*}{$p$ value } \\
\hline & A & $\mathrm{B}$ & & & \\
\hline $\mathrm{Ki}-67 \mathrm{LI} \pm \mathrm{SD}$ & $31.4 \pm 16.0$ & $39.0 \pm 21.4$ & 0.41 & & \\
\hline High LI* & $3(10.7 \%)$ & $13(61.9 \%)$ & 0.02 & 48.4 & 0.01 \\
\hline Low LI & $25(89.3 \%)$ & $8(38.1 \%)$ & & 30.5 & \\
\hline HPA & $15(53.6 \%)$ & $18(85.7 \%)$ & 0.04 & 38.0 & 0.08 \\
\hline Non-H PA & $13(46.4 \%)$ & $3(14.3 \%)$ & & 27.3 & \\
\hline \multicolumn{6}{|l|}{ Phenotypic classification } \\
\hline I type & $19(67.9 \%)$ & $8(34.8 \%)$ & $* *$ & & \\
\hline GI type & $4(14.3 \%)$ & $5(21.7 \%)$ & & & \\
\hline G type & $1(3.6 \%)$ & $7(30.4 \%)$ & $* * *$ & & \\
\hline $\mathrm{N}$ type & $4(14.3 \%)$ & $3(13.0 \%)$ & & & \\
\hline \multicolumn{6}{|l|}{ Number of TAMs } \\
\hline Minimum & 5 & 15 & & & \\
\hline Maximum & 68 & 81 & & & \\
\hline Median & 18 & 52 & & & \\
\hline Mean \pm SD & $21.8 \pm 14.3$ & $49.9 \pm 16.4$ & $<0.0001$ & & \\
\hline Number per $1 \mathrm{~mm}^{2}($ mean $\pm S D)$ & $332.4 \pm 206.0$ & $767.2 \pm 323.8$ & $<0.0001$ & & \\
\hline Areas of stroma of adenoma $\left(\times 10^{4} \mathrm{gm}^{2}\right)$ & 6.95 & 6.92 & 0.96 & & \\
\hline
\end{tabular}

Statistically significant values are shown in bold

$H P A$ highly proliferative adenoma, $I$ intestinal, $G I$ gastric and intestinal mixed, $G$ gastric, $N$ unclassified, TAM tumor-associated macrophage

* Ki-67 labeling index $>50 \%$

$* * p=0.03$ (I type vs. GI, G and N type)

$* * * p=0.02$ (G type vs. I, GI and $\mathrm{N}$ type)

95, respectively. High-grade atypia emerged at 133 months. The TAM number was related with the Ki-67 LI of adenomas ( $p=0.01$; Table 2$)$, indicating a mutual interaction between TAMs of stroma and the proliferative activity of adenoma cells, which was a novel finding.

\section{Discussion}

Gastric adenoma is regarded as a benign epithelial tumor and thought to possess a low malignant potential [31]. However, some adenomas progress into adenocarcinomas within a short time period, whereas others remain unchanged for a long time [8]. Although several groups have put forward important factors that are correlated with the development of gastric adenocarcinoma from adenoma, the definite factors that lead to a transformation to adenocarcinoma are still unclear. In the present study, two group A adenomas and all group B adenomas developed adenocarcinoma. We demonstrated the risk factors of group B adenomas that harbor synchronous adenocarcinoma or that transform to adenocarcinoma in a short time period. To our knowledge, the follow-up period of our series (mean 8.8 years, group A) is the longest compared to those of previous reports. Concerning the macroscopic findings, the presence of a depressed lesion and tumor size were correlated with malignant transformation of adenomas, consistent with previous studies [32]. In the microscopic findings, the presence of moderate or severe atypia, not severe atypia only, was evaluated as an independent risk factor by multivariate analysis in the present study. This is a novel finding and may emphasize the importance of performing an accurate morphological analysis.

Limitations of our study include the fact that we examined small biopsy specimens and almost all cases diagnosed as adenocarcinoma had an additional operation in another hospital, which makes it difficult to indicate the detailed distribution and depth of invasion. Mori et al., studying adenocarcinomas that emerged from adenoma with more than 3 years of follow-up, showed that all the resected lesions were intramucosal adenocarcinomas [33]. In the present study, resected lesions of group A (A4 and A9) were also intramucosal adenocarcinomas.

Several lines of evidence have suggested that the mutations of TP53 and CTNNB1 genes contribute to gastric carcinogenesis [34, 35]. A small proportion of gastric carcinomas may be caused by a specific germline mutation of the $C D H 1$ gene. In the present study, scattered nuclear staining of p53 was found in most adenomas regardless of the group. There were no significant differences in the p53, 

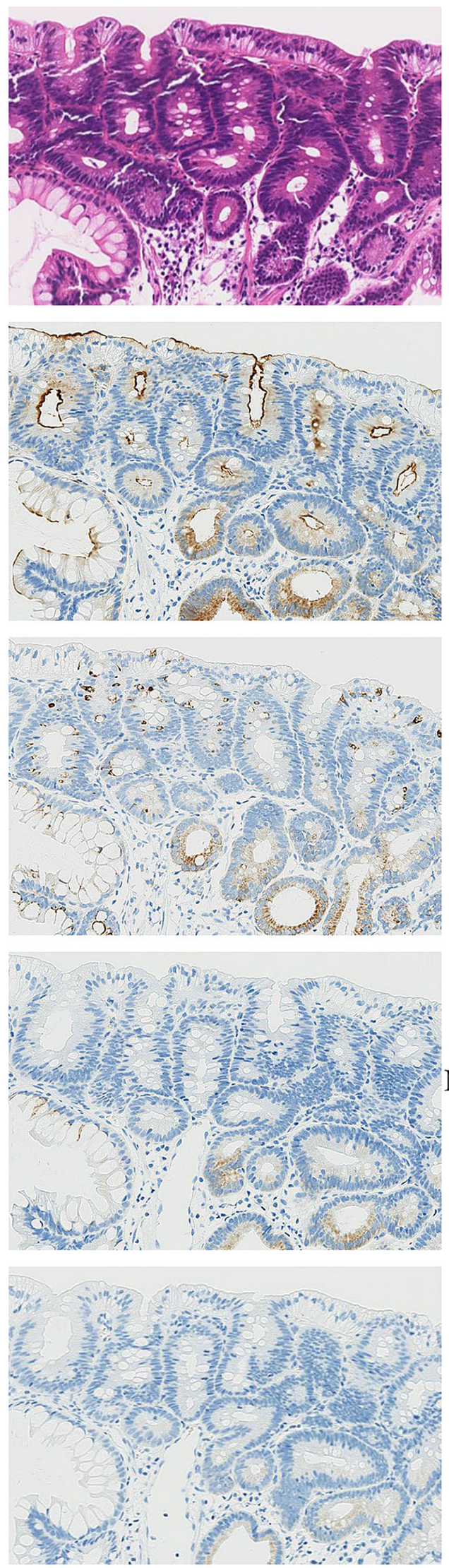

$\mathrm{HE}$
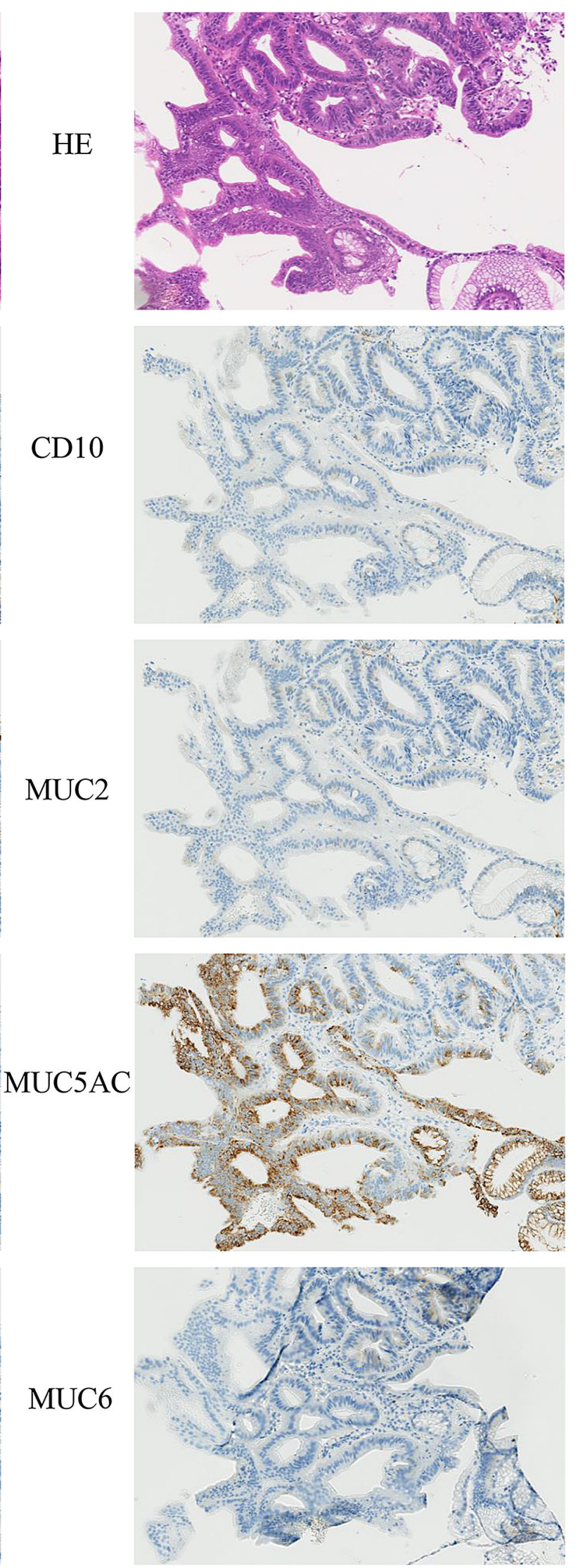
4Fig. 3 Two cases of low-grade adenomas, intestinal type (A14, left) and gastric type (A11, right). (Left) CD10 is expressed in the luminar surface along the apical membrane of adenomatous glands, and MUC2 is expressed in the goblet cells. (Right) MUC5AC is positive for the tumor cells

ALDH1, CD44 or Olfactomedin 4 IHC between the two groups (data not shown). However, our results revealed the significance of the distribution of proliferative cells, not LI itself [36]. Katakura et al. reported that Ki-67 positivity in surface epithelium was significantly higher in intramucosal carcinomas compared to adenomas in resected specimens [32]. They indicated the loss of polarity of proliferating cells in adenocarcinomas. In the present study, highly proliferative glands may indicate the loss of polarity of proliferating cells and they were found more frequently in group B. Therefore, adenomas with a loss of polarity of proliferating cells could favor carcinoma development. Further studies should be performed in the near future to
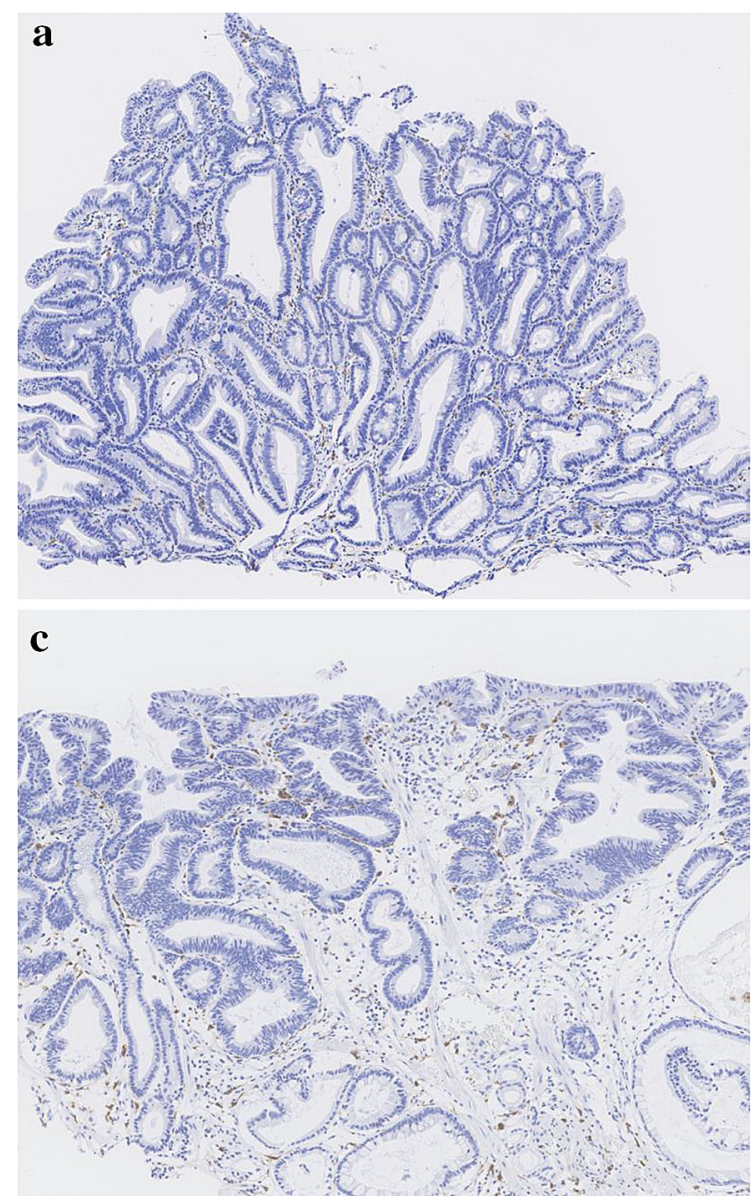

Fig. 4 Immunohistochemical analysis of CD204. CD204-positive TAMs were counted in the adenoma stroma in three high-power microscopic fields. a, b Low- and high-power view of A9 adenoma with mild nuclear atypia. Only a few CD204-positive TAMs in the elucidate the role of several genes such as CTNNB1 and $\mathrm{CDH} 1$ in gastric adenoma.

Gastric adenomas have been known to predominantly express an I phenotype, whereas adenocarcinomas predominantly express a $G$ phenotype. Nishimura et al. reported that low-grade adenomas with I phenotype may progress to high-grade adenomas, but rarely become intramucosal adenocarcinoma [36]. In the present study, group A adenomas showed the I phenotype more frequently, but group B adenomas showed the $G$ phenotype more frequently than the opposite group adenomas. Two group A adenomas that developed adenocarcinoma after a long follow-up showed the I phenotype at the initial biopsy. These data may suggest that low-grade adenomas with the I phenotype have a low potential to progress to adenocarcinoma.

There have been no reports regarding the significance of TAMs in gastric adenoma. We found that the TAM numbers in the stroma of adenoma were significantly larger in group $\mathrm{B}$, and there was a significant correlation between
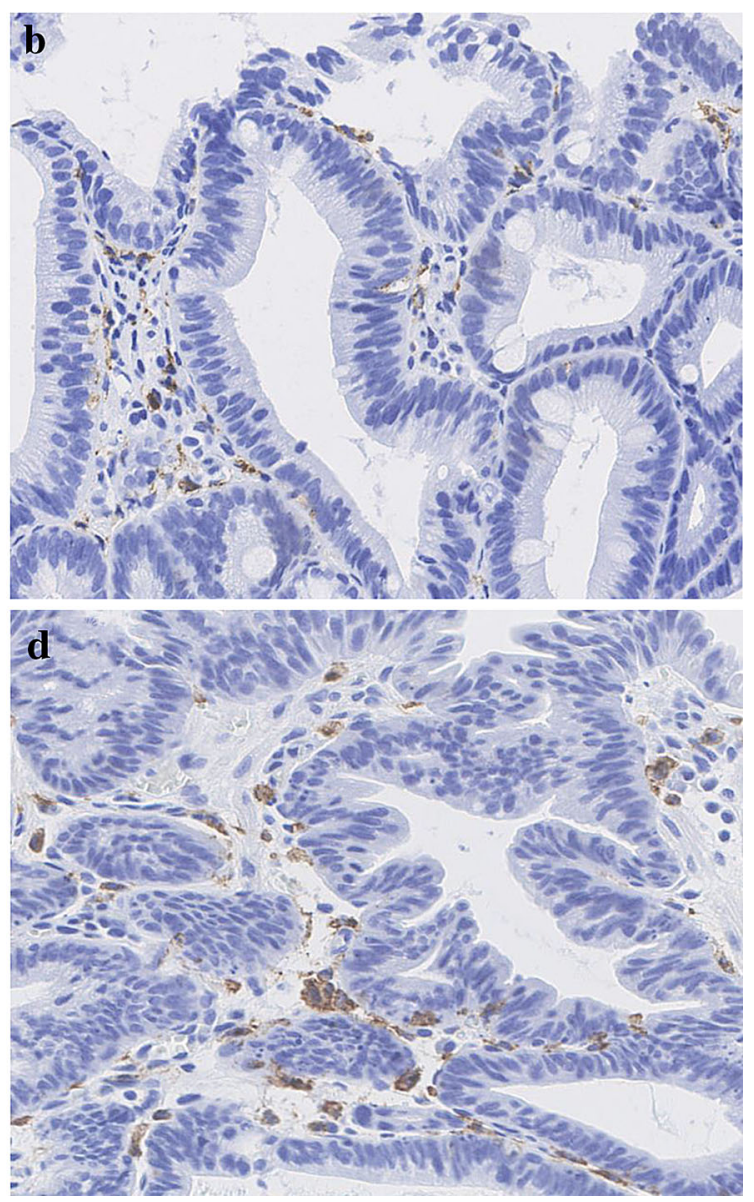

adenoma stroma are detected. c, d Low- and high-power view of B13 adenoma with severe nuclear atypia. Many CD204-positive TAMs in the adenoma stroma are detected 
Table 3 Univariate and multivariate analysis of factors for adenocarcinoma

\begin{tabular}{|c|c|c|c|c|c|c|}
\hline & \multicolumn{3}{|c|}{ Univariate analysis } & \multicolumn{3}{|c|}{ Multivariate analysis } \\
\hline & OR & $95 \% \mathrm{CI}$ & $p$ value & OR & $95 \% \mathrm{CI}$ & $p$ value \\
\hline \multicolumn{7}{|l|}{ Tumor size } \\
\hline$<10 \mathrm{~mm}$ & 1 & & & 1 & & \\
\hline$\geq 10 \mathrm{~mm}$ & 3.96 & $10,230-12.734$ & 0.02 & 1.03 & $0.108-8.723$ & 0.99 \\
\hline \multicolumn{7}{|l|}{ Depressed lesion } \\
\hline Absent & 1 & & & 1 & & \\
\hline Present & 5.69 & $1.049-30.835$ & 0.03 & 1.03 & $0.001-2.686$ & 0.18 \\
\hline \multicolumn{7}{|l|}{ Ki-67 LI } \\
\hline Low $(<50 \%)$ & 1 & & & & & \\
\hline High $(\geqq 50 \%)$ & 5.13 & $1.160-22.676$ & 0.02 & 0.306 & $0.008-5.835$ & 0.44 \\
\hline \multicolumn{7}{|l|}{ Distribution } \\
\hline Non-HPA & 1 & & & 1 & & \\
\hline HPA & 5.2 & $1.244-21.733$ & 0.01 & 3.378 & $0.253-69.221$ & 0.4 \\
\hline \multicolumn{7}{|l|}{ Phonotype } \\
\hline Others (GI, G and N) & 1 & & & & & \\
\hline I type & 0.27 & $0.083-0.878$ & 0.03 & 1.309 & $0.085-28.055$ & 0.85 \\
\hline Others (GI, G and N) & 1 & & & & & \\
\hline G type & 10.13 & $1.116-91.88$ & 0.02 & 0.312 & $0.005-13.427$ & 0.52 \\
\hline \multicolumn{7}{|l|}{ Atypia } \\
\hline Mild & 1 & & & & & \\
\hline Moderate or severe & 28.5 & $6.290-129.13$ & $<0.01$ & 11.51 & $1.706-445.81$ & 0.01 \\
\hline \multicolumn{7}{|l|}{ Number of TAM } \\
\hline Low (<medium) & 1 & & & 1 & & \\
\hline High ( $\geqq$ medium $)$ & 36.7 & $6.625-202.94$ & $<0.01$ & 65.35 & $4.764-3489.6$ & $<0.01$ \\
\hline
\end{tabular}

Statistically significant values are shown in bold

$L I$ labeling index, HPA highly proliferative adenoma, $I$ intestinal, $G I$ gastric and intestinal mixed, $G$ gastric, $N$ unclassified, TAM tumor-associated macrophage

$N=51, \mathrm{R} 2=0.61$, Model $p$ value $: p<0.0001$
TAM numbers and the proliferating activity of adenoma cells. These findings may indicate that TAMs and adenoma cells interact with each other in carcinoma development of gastric adenoma. The TAM number, not only moderate or severe atypia, was evaluated as an independent risk factor by multivariate analysis (Table 3 ). In A4 and A9 adenomas, TAM numbers tended to increase gradually during follow-up. These findings indicate that the TAM function may precede the morphological change of adenoma cells.

In this study, obvious differences were demonstrated between group A and group B except for age at the first biopsy, which supports the perception that some differentiated gastric adenocarcinomas may not develop through the sequence from group A to group B. We conclude that harboring or progressing to adenocarcinoma in a short time could be suggested macroscopically by the tumor size and presence of a depressed lesion of the adenoma. The presence of a gastric phenotype, moderate or severe atypia, and TAM numbers are critical microscopic findings that suggest carcinoma development regardless of the follow-up duration. Detecting these factors alerts a patient to the high possibility of carcinoma and to undergo the necessary treatment. The significance of TAMs in an adenoma-carcinoma sequence is a novel finding, and the role of TAMs in carcinoma development should be further elucidated.

Acknowledgements The authors would like to thank Mitsuki Nishimura, Akiko Ishimoto, Mayumi Takeuchi and Yoko Kodama for their excellent technical assistance and organization of this research. This work was supported financially in part by the National Hospital Organization of Japan.

\section{Compliance with ethical standards}

Conflict of interest The authors declare no conflicts of interest.

Human rights statement and informed consent All procedures followed were in accordance with the ethical standards of the responsible committee on human experimentation (institutional and national) and with the Helsinki Declaration of 1964 and later versions. Informed consent or a substitute for it was obtained from all patients for being included in the study. 


\section{References}

1. Goldstein NS, Lewin KJ. Gastric epithelial dysplasia and adenoma: historical review and histological criteria for grading. Hum Pathol. 1997;28:127-33.

2. Park do Y, Lauwers GY. Gastric polyps: classification and management. Arch Pathol Lab Med. 2008;132:633-40.

3. Stolte M, Sticht T, Eidt S, Ebert D, Finkenzeller G. Frequency, location, and age and sex distribution of various types of gastric polyp. Endoscopy. 1994;26:659-65.

4. Nakamura T, Nakano G. Histopathological classification and malignant change in gastric polyps. $J$ Clin Pathol. 1985;38:754-64.

5. Hattori T. Morphological range of hyperplastic polyps and carcinomas arising in hyperplastic polyps of the stomach. J Clin Pathol. 1985;38:622-30.

6. Goddard AF, Badreldin R, Pritchard DM, Walker MM, Warren B. The management of gastric polyps. Gut. 2010;59:1270-6.

7. Tsukashita S, Kushima R, Bamba M, Sugihara H, Hattori T. MUC gene expression and histogenesis of adenocarcinoma of the stomach. Int J Cancer. 2001;94:166-70.

8. Miwa K, Doyama H, Hirano K, Inagaki S, Waseda Y, Goto Y, et al. Is endoscopic differential diagnosis possible between adenoma and adenocarcinoma in the cases with biopsy-based preoperative diagnosis of gastric adenoma treated by endoscopic submucosal dissection? Endosc Forum Dig Dis. 2010;26:17-26.

9. Lauwers GY, Riddell RH. Gastric epithelial dysplasia. Gut. 1999;45:784-90.

10. Lansdown M, Quirke P, Dixon MF, Axon AT, Johnston D. High grade dysplasia of the gastric mucosa: a marker for gastric carcinoma. Gut. 1990;31:977-83.

11. Kolodziejczyk P, Yao T, Oya M, Nakamura S, Utsunomiya T, Ishikawa T, et al. Long-term follow-up study of patients with gastric adenomas with malignant transformation. An immunohistochemical and histochemical analysis. Cancer. 1994;74:2896-907.

12. Yamada H, Ikegami M, Shimoda T, Takagi N, Maruyama M. Long-term follow-up study of gastric adenoma/dysplasia. Endoscopy. 2004;36:390-6.

13. Sung JK. Diagnosis and management of gastric dysplasia. Korean J Intern Med. 2016;31:201-9.

14. Di Gregorio C, Morandi P, Fante R, De Gaetani C. Gastric dysplasia. A follow-up study. Am J Gastroenterol. 1993;88:1714-9.

15. Rugge M, Farinati F, Baffa R, Sonego F, Di Mario F, Leandro G, et al. Gastric epithelial dysplasia in the natural history of gastric cancer: a multicenter prospective follow-up study. Interdisciplinary Group on Gastric Epithelial Dysplasia. Gastroenterology. 1994;107:1288-96.

16. Saraga EP, Gardiol D, Costa J. Gastric dysplasia. A histological follow-up study. Am J Surg Pathol. 1987;11:788-96.

17. Fertitta AM, Comin U, Terruzzi V, Minoli G, Zambelli A, Cannatelli $G$, et al. Clinical significance of gastric dysplasia: a multicenter follow-up study. Gastrointestinal Endoscopic Pathology Study Group. Endoscopy. 1993;25:265-8.

18. Kokkola A, Haapiainen R, Laxen F, Puolakkainen P, Kivilaakso E, Virtamo J, et al. Risk of gastric carcinoma in patients with mucosal dysplasia associated with atrophic gastritis: a follow up study. J Clin Pathol. 1996;49:979-84.

19. Visvader JE, Lindeman GJ. Cancer stem cells in solid tumours: accumulating evidence and unresolved questions. Nat Rev Cancer. 2008;8:755-68.

20. Mantovani A, Sozzani S, Locati M, Allavena P, Sica A. Macrophage polarization: tumor-associated macrophages as a paradigm for polarized M2 mononuclear phagocytes. Trends Immunol. 2002;23:549-55.
21. Sica A, Schioppa T, Mantovani A, Allavena P. Tumour-associated macrophages are a distinct M2 polarised population promoting tumour progression: potential targets of anti-cancer therapy. Euro J Cancer (Oxford, England: 1990). 2006;42:717-27.

22. Tsutsui S, Yasuda K, Suzuki K, Tahara K, Higashi H, Era S. Macrophage infiltration and its prognostic implications in breast cancer: the relationship with VEGF expression and microvessel density. Oncol Rep. 2005;14:425-31.

23. Ohtaki Y, Ishii G, Nagai K, Ashimine S, Kuwata T, Hishida T, et al. Stromal macrophage expressing CD204 is associated with tumor aggressiveness in lung adenocarcinoma. J Thorac Oncol Off Publ Int Assoc Study Lung Cancer. 2010;5:1507-15.

24. Lissbrant IF, Stattin P, Wikstrom P, Damber JE, Egevad L, Bergh A. Tumor associated macrophages in human prostate cancer: relation to clinicopathological variables and survival. Int J Oncol. 2000; $17: 445-51$.

25. Yoshikawa K, Mitsunaga S, Kinoshita T, Konishi M, Takahashi $\mathrm{S}$, Gotohda N, et al. Impact of tumor-associated macrophages on invasive ductal carcinoma of the pancreas head. Cancer Sci. 2012;103:2012-20.

26. Sano T, Aiko T. New Japanese classifications and treatment guidelines for gastric cancer: revision concepts and major revised points. Gastric Cancer Off J Int Gastric Cancer Assoc Jpn Gastric Cancer Assoc. 2011;14:97-100.

27. Schlemper RJ, Riddell RH, Kato Y, Borchard F, Cooper HS, Dawsey SM, et al. The Vienna classification of gastrointestinal epithelial neoplasia. Gut. 2000;47:251-5.

28. Taniyama K, Ishida K, Toda T, Motoshita J, Kuraoka K, Saito A, et al. Tyrosine1248-phosphorylated HER2 expression and HER2 gene amplification in female invasive ductal carcinomas. Breast cancer (Tokyo, Japan). 2008;15:231-40.

29. Oue N, Sentani K, Noguchi T, Ohara S, Sakamoto N, Hayashi T, et al. Serum olfactomedin 4 (GW112, hGC-1) in combination with Reg IV is a highly sensitive biomarker for gastric cancer patients. Int J Cancer. 2009;125:2383-92.

30. Aung PP, Oue N, Mitani Y, Nakayama H, Yoshida K, Noguchi T, et al. Systematic search for gastric cancer-specific genes based on SAGE data: melanoma inhibitory activity and matrix metalloproteinase-10 are novel prognostic factors in patients with gastric cancer. Oncogene. 2006;25:2546-57.

31. Nagatomi Y, Kawamura S, Kawashima M, Harima K, Maetani N, Azuma M, et al. Clinical follow-up of protruded atypical epithelial foci of the stomach. Gastroenterol Endosc. 1980;22:275-83_1.

32. Katakura T, Masuda T, Ikeda T, Abe S, Shimada T, Shibuki S, et al. Immunohistochemical studies in depressed type adenomas of the stomach-comparison with protruded type adenomas and depressed type well differentiated mucosal gastric cancers. Nihon Shokakibyo Gakkai zasshi=. Jpn J Gastro-Enterol. 1998;95:992-1000.

33. Mori G, Oda I, Taniguchi H, Miyamoto Y, Sekiguchi M, Abe S, et al. Endoscopic surveillance for clinical management of gastric adenoma. Stomach Intestine. 2014;49:1859-69.

34. Jang B-G, Kim WH. Molecular pathology of gastric carcinoma. Pathobiology. 2011;78:302-10.

35. Fenoglio-Preiser C, Wang J, Stemmermann G, Noffsinger A. TP53 and gastric carcinoma: a review. Hum Mutat. $2003 ; 21: 258-70$.

36. Nishimura R, Mukaisho K, Yamamoto H, Sonoda A, Andoh A, Fujiyama Y, et al. Precursor-derived versus de-novo carcinogenesis depends on lineage-specific mucin phenotypes of intramucosal gland-forming gastric neoplasms. Histopathology. 2013;63:616-29. 